\title{
A MONTE CARLO-FINITE ELEMENT MODEL FOR STRAIN ENERGY CONTROLLED MICROSTRUCTURAL EVOLUTION: "RAFTING" IN SUPERALLOYS
}

\section{J. GAYDA ${ }^{1}$ and D. J. SROLOVITZ ${ }^{2}$}

'National Aeronautics and Space Administration, Lewis Research Center, Cleveland, $\mathrm{OH} 44135$ and ${ }^{2}$ Department of Materials Science and Engineering, University of Michigan, Ann Arbor, MI 48109, U.S.A.

\author{
(Received 18 May 1988)
}

\begin{abstract}
A specialized microstructural model, MCFET, has been developed which simulates microstructural evolution in materials in which strain energy plays an important role in determining morphology. The elastic contribution to the energy of the system is estimated by way of a finite element procedure which is employed in a Monte Carlo procedure to determine the microstructural evolution. This model is currently capable of accounting for externally applied stresses, surface tension, misfit, elastic inhomogeneity, elastic anisotropies (cubic) and arbitrary temperatures. The MCFET analysis has been validated by comparison with analytical calculations of the equilibrium morphologies of isolated particles in an infinite matrix. Larger simulations yield microstructures which are in good agreement with experimental observations (including the influence of applied stress). Simulations on the coarsening of two-phase microstructures containing misfitting particles suggest that the presence of strong elastic interactions greatly decreases the rate of microstructural coarsening.
\end{abstract}

\begin{abstract}
Résumé-Un modèle microstructural particulier, MCFET, a été développé pour simuler l'évolution microstructural de matériau où l'énergie de déformation joue un rôle important dans la détermination de la morphologie. La contribution élastique à l'énergie du système est estimée à l'aide d'une méthode d'éléments finis que l'on utilise dans un calcul de Monte Carlo pour déterminer l'évolution microstructurale. Ce modèle peut couramment tenir compte des contraintes externes appliquées, de la tension superficielle, du désaccord, de l'hétérogénéité élastique, des anisotropies élastiques (cubiques) et de la température. Cette analyse MFCET a été validée en la comparant avec les calculs analytiques de morphologies à l'équilibre de particules isolées dans une matrice infinie. Des simulations plus poussées conduisent à des microstructures qui sont en bon accord avec l'expérience (y compris pour l'infiuence de la contrainte appliquée). Les simulations du grossissement de microstructures biphasées contenant des particules incohérentes suggèrent que la présence de fortes interactions élastiques provoque une forte diminution de la vitesse du grossissement microstructural.
\end{abstract}

\begin{abstract}
Zusammenfassung - Ein spezielles Mikrostrukturmodell, MCFET, wurde entwicklet, mit dem die Entwicklung der Mikrostruktur in einem Material, in dem die Verzerrungsenergie im wesentlichen die Morphologie bestimmt, simuliert werden kann. Der elastische Beitrag zur Energie des Systems wird mittels eines Verfahrens der finiten Elemente abgeschätzt; dieses Verfahren wird in dem Rahmen des MonteCarlo-Verfahrens zur Bestimmung der Entwicklung der Mikrostruktur eingesetzt. Das Modell kann gegenwärtig die äußere Last, Oberflächenspannungen, Fehlpassung, elastische Inhomogenität, elastische Anisotropien und beliebige Temperaturen berücksichtigen. Die MCFET-Analyse wurde verifiziert mit einem Vergleich mit analytischen Berechnungen der Gleichgewichtsformen isolierter Teilchen in einer undenlichen Matrix. Ausgedehntere Simulationen ergeben Mikrostrukturen, die mit experimentellen Beobachtungen (einschließlich des Einflusses der äußeren Spannung) übereinstimmen. Simulation der Vergröberung einer zweiphasigen Mikrostruktur, die fehlpassende Teilchen enthält, legen die Annahme nahe, daß starke elastische Wechselwirkungen die Rate der mikrostrukturellen Vergröberung stark verlangsamen.
\end{abstract}

\section{INTRODUCTION}

Since many of the physical properties of materials are determined by microstructure, it is important to be able to predict and control microstructural development. This is especially true and increasingly difficult when the microstructure consists of multiple phases. Further complexity is added when there is strong coupling between the external environment and the internal microstructure: for example, due to elastic interactions. Recently, a microstructural lat- tice simulation technique has been developed which accounts for microstructural evolution in relatively simple systems $[1,2]$. Such techniques can, in principle, incorporate essentially all relevant driving forces and kinetic considerations into a single simulation [3]. The present study extends this computer simulation technique to cases in which the microstructural evolution is controlled, in part, by strain energy. This is accomplished by coupling a Monte Carlo procedure with finite element calculations of the strain energy. This technique is applied to the 
coarsening and rafting of the precipitates in a Ni-based $\gamma-\gamma^{\prime}$ superalloy.

The essential elements of this approach consist of mapping the continuum microstructure onto a discrete lattice and defining interactions and dynamics for the lattice points which are analogous to those in a continuous system. Each site is assigned a label which corresponds to a particular phase (e.g. $\gamma^{\prime}$ or $\gamma$, in the Ni-based superalloys). In cases where the quantity of each phase does not evolve with time, a Monte Carlo procedure with conserved dynamics [4] will be employed. This Monte Carlo procedure chooses a pair of neighboring sites at random, attempts to exchange them, and either accepts or rejects the exchange based on the associated energy change of the system. Since exchanges between adjacent sites are attempted at random, this procedure corresponds to diffusion controlled microstructural evolution. The energetics of the system are determined by the competition between surface tension and elastic interactions. The elastic interactions account for the misfit between the $\gamma$ and $\gamma^{\prime}$ phases, their different elastic moduli, the shapes of the precipitates, and the effects of external stress fields.

Since this Monte Carlo Finite Element Technique (MCFET) is new, the method is described in detail, and comparisons between its predictions and simple analytical theory are made. In the latter half of the paper, MCFET is applied to the case of coarsening and rafting of the $\gamma-\gamma^{\prime}$ microstructure in the presence of an externally applied tensile stress, an externally applied compressive stress, and under no external loading.

\section{MCFET APPROACH}

In the MCFET microstructural modeling, we employ an $n \times n\left(n^{2}=N\right)$ two dimensional square lattice. Each lattice site (microstructural element) is associated with either the precipitate phase, $P$, or the matrix phase, $M$, as shown in Fig. 1 . The Monte

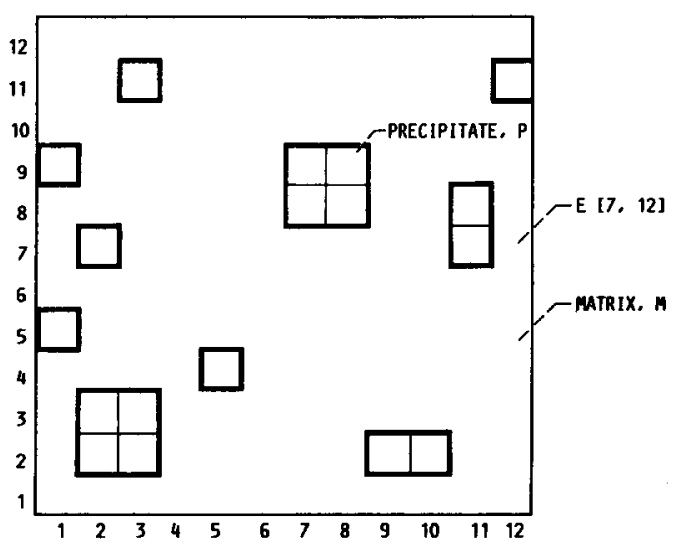

Fig. 1. The square grid employed in the Monte Carlo simulations. Each site is associated with a label $(P$ or an $M)$ to indicate the phase associated with its environment.
Carlo procedure consists of the following steps: (1) choose one of the $N$ lattice sites at random, (2) choose one of its nearest or next-nearest neighbors at random, (3) calculate the energy of the system in this configuration, (4) exchange the two sites, (5) recalculate the energy of the system, (6) calculate the probability, $P(\Delta E)$, that this exchange will be accepted or rejected, (7) generate a random number, $R$ $(1 \geqslant R \geqslant 0)$, (8) accept the exchange if and only if $R<P$, (9) return to step one. For a simulation containing $N$ lattice sites, $N$ exchange attempts are referred to as 1 Monte Carlo Step (MCS). The exchange procedure corresponds to a simple rate theory description of diffusion in which the Monte Carlo Step is the unit of time. Unfortunately, this identity is uncertain to within a multiplicative constant since the activation energy for diffusion has been scaled out of the problem.

The energy of the system is, in principle, given by

$$
E^{\text {total }}=\sum_{i=1}^{N}\left\{E_{i}^{\text {elastic }}+(1 / 2) \sum_{j} J\left(1-\delta_{s_{i} s_{j}}\right)\right\}
$$

where $E_{i}^{\text {elastic }}$ is the strain energy associated with site $i, s_{i}$ and $s_{j}$ are the phases associated with site $i$ and $j$, respectively, $\delta$ is the Kronecker delta function, $J$ is a positive constant ( $J$ divided by the lattice spacing is the surface tension for the 2-dimensional system), and the second summation is taken over nearest neighbors of site $i$. The second term in equation (1) accounts for the precipitate/matrix interfacial energy. The first term accounts for the elastic energy of the system and will be discussed, in detail, in the following section. It should be noted that while equation (1) gives the total energy of the system, the only energetics that enter the Monte Carlo simulation is the difference in energy between the configurations before and after the attempted exchange. The numerical value of the constants entering equation (1) explicitly or implicitly within the elastic energy term are discussed below.

The probability that a certain exchange, associated with an energy change $\Delta E$, is accepted or rejected is

$$
P=(1 / 2)[1-\tanh (\Delta E / 2 k T)]
$$

where $k T$ is the thermal energy. This is the standard probability expression employed in conserved dynamics [4]. In practice, equation (2) is employed in the Monte Carlo procedure by generating a random number, $R$ (uniformly distributed between 0 and 1 ), and accepting the exchange if $R<P$ and, otherwise, rejecting it.

Periodic boundary conditions have heen employed in the present simulations to minimize the effect of the finite lattice size. The simulations described in the latter half of this paper were all performed on square lattices containing $N=2500$ sites $(50 \times 50)$. In order to improve the statistics on the $50 \times 50$ simulations, the data reported were averaged over at least 5 simulations. The simulations which were performed 
on these large lattices were initiated by assuming a random solid solution of $P$ sites in a background of $M$ sites.

\section{FINITE ELEMENT STRESS ANALYSIS}

Although finite element techniques can be applied to the present problem without great difficulty, the enormous number of exchanges to be considered in a realistic analysis would require an impractically large amount of computer time to calculate the mechanical energy over the entire Monte Carlo lattice for each exchange. Therefore, one must devise a scheme which limits the computation time per exchange. In the present analysis this is accomplished by confining the stress analysis to a small region centered about the sites to be exchanged and calculate the mechanical energy of this smaller, local grid before and after each exchange. While the absolute energies will be affected by this cut-off in the stress analysis, the difference in energies between the two local configurations will be small. The relatively small error this cut-off induces in $\Delta E$ will be quantified in the following section.

The smaller, local grid upon which the stress analysis is performed is shown in Fig. 2. It is generated from the larger, global Monte Carlo lattice and is seen to contain 36 elements with the exchange pair residing in the 4 central sites. The actual element type used is the two-dimensional, simplex triangle and, as shown in Fig. 2, each Monte Carlo lattice element contains two such simplex triangles. For this element type and grid size, each energy calculations must set-up and solve a system of linear equations containing 98 unknowns. Since such solutions can be found relatively quickly it is possible to perform MCFET analyses which require of order $10^{5}-10^{6}$ such elastic energy calculations.

The elastic properties, modulus, Poisson's ratio, etc. of each element are assigned values corresponding to the phase identity of that element. To model misfit strains between precipitate and matrix, a volu-

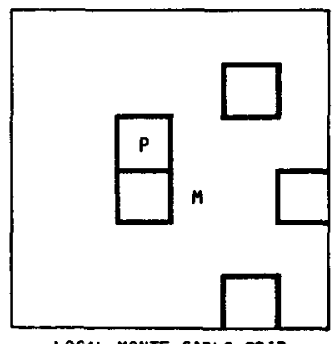

LOCAL MONTE CARLO GRID

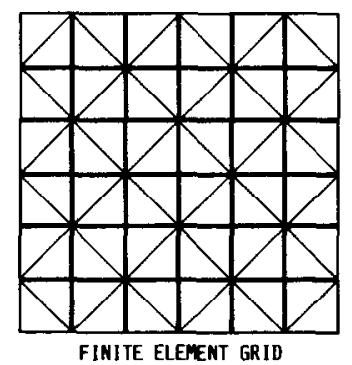

FINITE ELEMENT GRID
Fig. 2. The local Monte Carlo and finite element grids which are subsets of the Monte Carlo grid schematically illustrated in Fig. 1. Note the central location of the $P-M$ exchange pair $(E[7,12]$ and $E[8,11]$ in the global grid) in the local grid. The finite element grid is composed of two-dimensional simplex triangles. metric strain is simulated by replacing the usual thermal strain term in the finite element formulation with a numerically equivalent misfit strain. For a two phase analysis in which the misfit strain is $-0.1 \%$, the thermal strain term of the matrix elements are set equal to $0.05 \%$ while that for the precipitate elements is set equal to $-0.05 \%$.

The finite element code employed in the present analysis is simple but nevertheless time efficient, and was adopted from the text of Segerlind [5]. In this code the nodal displacements of the finite element grid are computed by minimizing the potential energy of the system for a given set of boundary conditions. From these nodal displacements the element stress and strains are calculated, and finally the elastic energy of the local grid ( $E_{i}^{\text {elastic }}$ ) is determined. The choice of boundary conditions are somewhat ambiguous in the present problem since the local finite element grid is supposed to correspond to a small section of an infinite solid. The boundary conditions employed eliminate rigid body motion by fixing the displacements of the central node at zero and eliminate rigid body rotation by setting the lateral displacement of the node directly above the central node at zero. Otherwise the body remains essentially unconstrained. Since the two sites being exchanged are always in the center of the local finite element grid, this procedure does not bias the exchange direction. For cases in which a uniaxial stress is applied to the system, loads of equal magnitude but of opposite sign are applied to the upper and lower boundary nodes of the finite element grid. These boundary conditions and the approximation introduced by the use of a relatively small grid size correspond to the assumption that stress fields associated with individual microstructural features decay rapidly. It is important to note, that since the elastic energy is calculated anew before and after each exchange, any errors made in the energy calculations are not accumulated.

The actual codes employed in the MCFET may be found in Ref. [6] In addition to these codes there is a complete description of the data employed as well as a simplified flowchart describing the overall approach.

\section{VALIDATION OF THE MCFET APPROACH}

Prior to analyzing more complex problems with multiple particle microstructures it is instructive to consider first some simplified microstructures containing one or two particles. In order to further simplify this analysis, we will initially ignore surface tension [i.e. $J=0$ in equation (1)], assume isotropic elasticity, and set $T=0$. This latter assumption does not imply that the system has zero diffusivity, instead it only allows for exchanges which decrease the energy of the system.

As discussed above, the size of the finite element grid must be smaller than the global Monte Carlo 
lattice for computational reasons. In order to choose the size of the finite element such as not to introduce large errors in $\Delta E$, a series of calculations were performed as a function of finite element grid size. These calculations were performed on a linear array of particles (each containing four sites arranged in a square) with a misfit of $-0.1 \%$, a particle/matrix modulus ratio of 0.8 , and an applied stress of $0.3 \mathrm{GPa}$ (see Fig. 3). Finite element grid sizes of $6 \times 6$, $12 \times 12$, and $18 \times 18$ were employed. While the total energy of the finite element grid clearly increases with grid size, this is largely attributable to the externally applied stress field and the increasing area of the grids. However, when normalized by the grid size, the variation in energy from the $6 \times 6$ to the $18 \times 18$ grid is of order $20 \%$. The change in energy corresponding to the exchange of next-nearest neighbor precipitate and matrix sites was also calculated. The change in energy associated with this exchange shows less than a $2 \%$ variation over the range of finite element grid size examined. Hence, it appears that the choice of a $6 \times 6$ finite element grid provides adequate accuracy and reasonable computation time.

The equilibrium shape of a particle depends on the relative elastic properties of the particle and matrix and on the magnitude and direction of the external stress field. This problem has been analyzed for ellipsoidal particles by Eshelby [7]. Pineau [8] derived

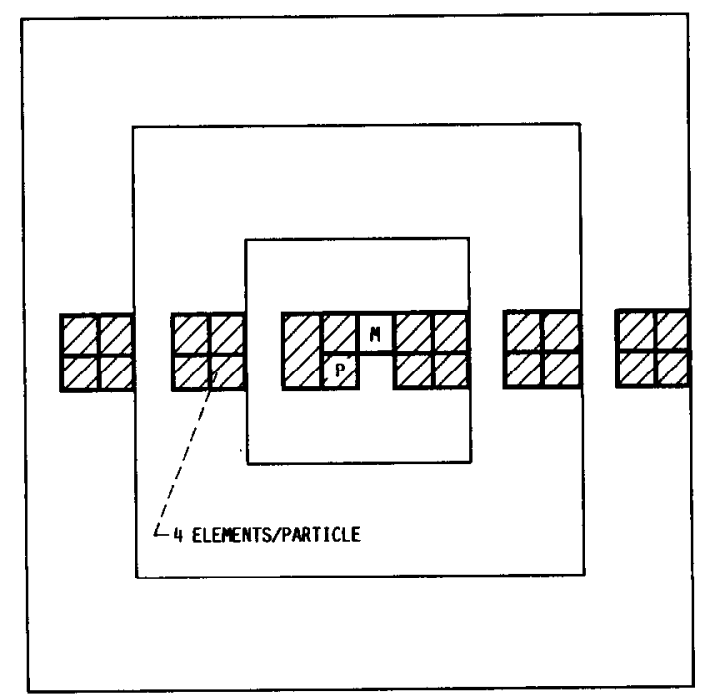

\begin{tabular}{|c|c|c|c|}
\hline $\begin{array}{c}\text { GRID SIZE } \\
(\mathbf{N})\end{array}$ & $\mathrm{E}_{\mathrm{N}^{\prime} / \mathrm{E}_{18}^{\prime}}$ & $\left(\mathrm{E}_{\mathbf{N}}^{\prime} / \mathrm{E}_{18}^{\prime}\right)(18 / \mathrm{N})^{2}$ & $\Delta \mathrm{E}_{\mathbb{N}} / \Delta \mathrm{E}_{18}$ \\
\hline 6 & 0.132 & 1.188 & 0.992 \\
12 & 0.464 & 1.044 & 0.981 \\
18 & 1.000 & 1.000 & 1.000 \\
\hline
\end{tabular}

Fig. 3. Calculation of the energies associated with a linear array of square particles. The elastic energies are calculated before and after the exchange of the sites laheled $P$ and $M$ on finite element grids of $6 \times 6,12 \times 12$ and $18 \times 18$. The data presented in the table is the energy of the $N \times N$ grids normalized by that of the $18 \times 18$ grid, the energy normalized to the area of the $18 \times 18$ grid, and the change in energy associated with the indicated site exchange.
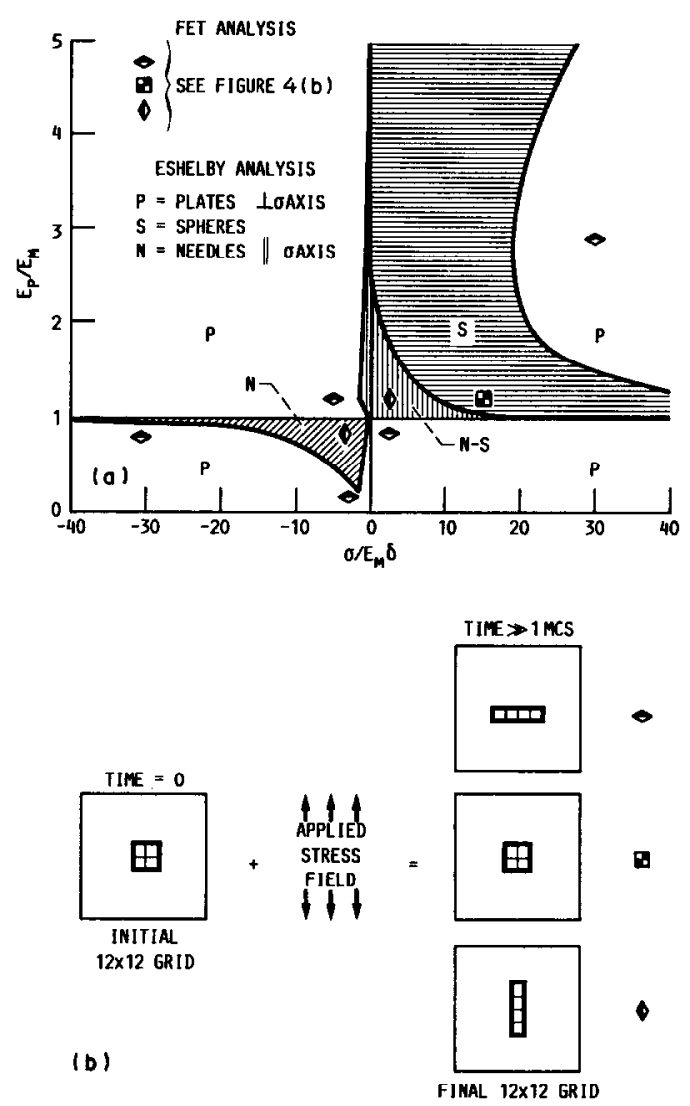

Fig. 4. (a) Comparison of the Monte Carlo-Finite Element Technique predictions of the stable particle shape with the elastic analysis of Pineau [8]. $E_{\mathrm{p}} / E_{\mathrm{m}}$ is the ratio of the particle to matrix moduli, $\sigma$ is the applied stress, and $\delta$ is the misfit. (b) Some of the details of the MCFET calculation.

a particle morphology map as a function of external stress and elastic parameters. This map [see Fig. 4(a)] plots the ratio of precipiate to matrix stiffness on the vertical axis and the ratio of the applied stress to misfit, normalized by the matrix stiffness on the horizontal axis. This figure shows that the application of stress can have three effects; the particle may remain symmetric, the particle may elongate in the direction parallel to the stress axis, or it may elongate in the direction orthogonal to the stress axis. Inset into this analytically derived map are symbols corresponding to single particle MCFET simulations. In these simulations, the initial structure contained a single particle ( 4 sites arranged in a square) and a $12 \times 12$ Monte Carlo lattice was employed. All of the simulations resulted in elongated particles, except one, in which the initially symmetric particle shape remained unchanged. Although the simulations were two-dimensional and the analytical results were derived for three-dimensional particles, the correspondence between the simulation and analytical results are perfect.

The analytical analysis of the particle shape and the previous simulation (Fig. 4) assume that the particle is isolated in an essentially infinite matrix. In 
most situations of interest, each precipitate particle feels the influence of many others. A simple method of gauging the importance of this effect is to study the morphology of a pair of particles as a function of their separation, $R_{\mathrm{p}}$. A continuum elastic analysis of this situation was performed by Johnson and Voorhees [9]. Figure 5 shows the resulting particle morphologies for a matrix modulus, precipitate modulus, and relative misfit of $10^{11} \mathrm{~Pa}, 8 \times 10^{10} \mathrm{~Pa}$, and $-0.1 \%$, respectively. Such a choice traverses Pineau's map, Fig. $4(\mathrm{a})$, at $E_{\mathrm{p}} / E_{\mathrm{m}}=0.8$. It is important that the interparticle separation influences the stress level at which the initial configuration becomes unstable. While the applied stress level at which the morphological transition occurs does indeed depend on the interparticle separation, the basic features of Pineau's map seem unaffected.

\section{CHOICE OF PARAMETERS}

The MCFET simulations described in the previous section were performed at zero temperature and with zero surface tension. As such, the exchange dynamics were based solely on strain energy effects. In order to include the effects of finite temperature and surface tension a number of constants must be defined. These include the following physically significant ratios: $\tau=k T / J$ and $\mu_{\mathrm{i}}=M_{\mathrm{i}} L / \gamma$, where $\gamma$ is the precipitate/matrix interfacial energy per unit area, the $M_{\mathrm{i}}$ are the $\mathrm{i}$ independent elastic constants and $L$ is the distance between lattice sites in the simulations. Since the simulation is designed to study micro-

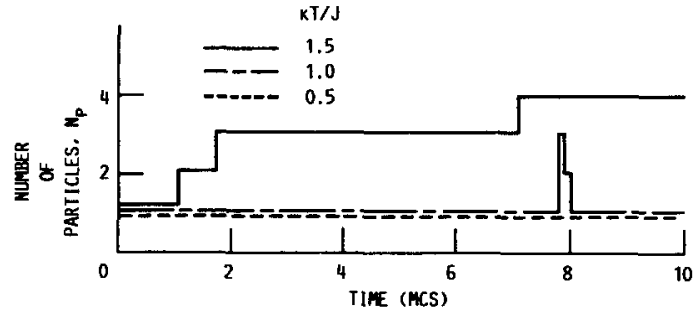

Fig. 6. The number of separate particles as a function of time at three different temperatures. The initial configuration was a single four site (arranged in a square) particle in a $12 \times 12$ matrix.

structural problems, we associate $L$ with a typical microstructural length scale, $10^{-6} \mathrm{~m}$. Further, we choose physically reasonable values for the other parameters, namely: the coherent precipitate/matrix interfacial energy is $0.02 \mathrm{~J} / \mathrm{m}^{2}$ and the elastic moduli of the particle and matrix are 80 and $100 \mathrm{GPa}$, respectively (Ref. [6] provides a description of the elastic constants employed).

The temperature at which the simulation will be performed was chosen to be below that at which a particle dissolves and sufficiently high that diffusion is relativcly fast. In order to identify this temperature, the evolution of a small particle (consisting of 4 particle sites arranged in a square; relative misfit $=-0.1 \%$; no external stress) was monitored for $\tau=0.5,1.0$ and 1.5. As seen in Fig. 6, at $\tau=0.5$ the particle neither broke up nor changed shape during this short run. At $\tau=1.5$, the particle broke
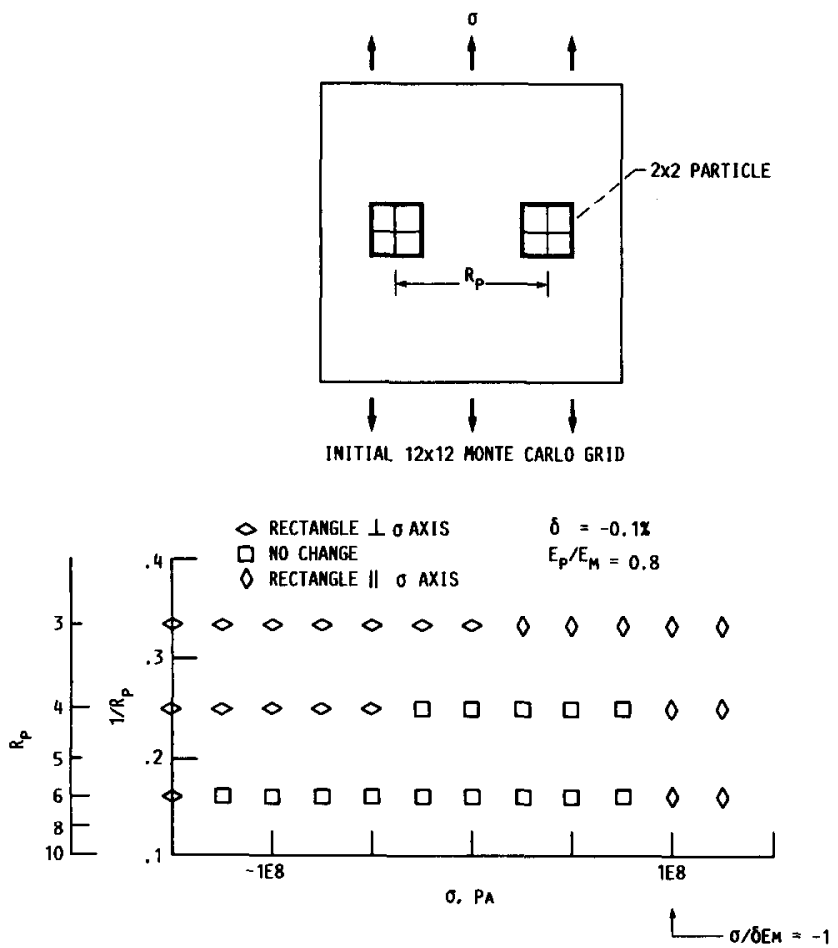

Fig. 5. The effect of interparticle separation, $R_{\mathrm{p}}$, on particle shape as a function of applied stress. 


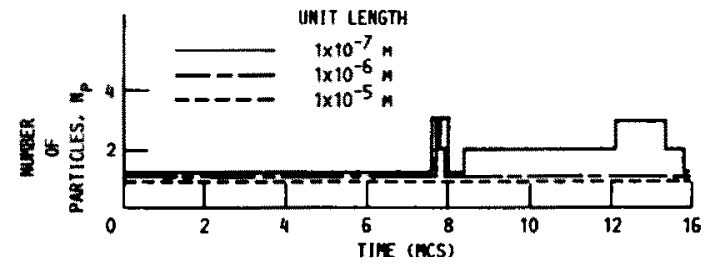

Fig. 7. As in Fig. 6, but at fixed temperature $(k T / J=1)$ but with different value of the unit length, $L$. See text for more details.

up completely within $7 \mathrm{MCS}$. Finally, at $\tau=1.0$, the particle momentarily breaks up and then recombines indicating the possibility for significant surface and bulk diffusion at this temperature. Based on these results, the majority of the simulations were performed at $\tau=1.0$.

In order to investigate simple particle size effects, three simulations were performed corresponding to choosing $L=10^{-5}, 10^{-6}$, and $10^{-7} \mathrm{~m}$ (all other conditions as above). As seen in Fig. 7, decreasing the size of the particle at fixed temperature decreases the particle stability. This result is not surprising since in the limit that the particle size is decreased below the critical nucleus size it must be unstable and hence, decays away. Since $L$ does not physically enter the problem as an independent parameter but only in terms of the $\mu_{\mathrm{j}}$, these results can likewise be interpreted in terms of $M$ or $\gamma$. In this sense, increasing $\gamma$ reduces the size of the critical nucleus and hence decreases the stability of the particle. Similarly, decreasing $M$ indicates decreasing binding within the particle and, therefore, decreasing particle stability.

\section{COARSENING OF 2-PHASE MICROSTRUCTURES}

A series of simulations were performed in which a two-phase microstructure was allowed to evolve with time under no load, under uniaxial compression and under uniaxial tension. The initial microstructures were generated at random and contained $40 \%$ precipitate phase and $60 \%$ matrix phase. Each $250 \mathrm{MCS}$ simulation on a $50 \times 50$ lattice required approximately $2 \mathrm{~h}$ of Cray-1s time, while a single $500 \mathrm{MCS}$ simulation on a $100 \times 100$ lattice required approximately $9 \mathrm{~h}$.

Figure 8 shows the temporal evolution of the two-phase microstructure for the case where no external load is applied. The initially random structure forms continuous networks of both the particle and matrix phases which clearly coarsens with time. Although these microstructures do not exhibit the ordered arrays of cuboidal precipitates often observed in Ni-based $\gamma-\gamma^{\prime}$ superalloys, nearly continuous, elongated precipitate morphologies, are also observed experimentally (see Ref. [12], for example). Figure 9 (b) shows the mean particle size as a function of time. While the mean particle size is clearly increasing with time, analysis of the data shows that it is growing more slowly than the expected $t^{1 / 3}$. This may be attributable to the elastic interactions between the misfitting precipitates, which competes with surface tension and tends to keep particle sizes small. Figure 9(a) shows the time dependence of the fraction of broken bonds (i.e. bonds between nearest neighbor sites of unlike phase) and the ratio of the total number of broken bonds in the $y$-direction to those in the $x$-direction. The total number of broken bonds decreases in time as the system coarsens, but after approximately $50 \mathrm{MCS}$ this decrease is extremely slow. Since there is no externally applied stress on the sample, the number of broken bonds in the $x$ and $y$ direction should be equal. This ratio is approximately equal to 1.25 in the present stimulation. The deviation of this parameter from unity provides an indication of our limited statistics in the present simulation (this data was averaged over 5 runs on $50 \times 50$ lattices).

When the applied stress is tensile, the precipate morphology is clearly elongated in the direction of the tensile axis (see Fig. 10). By 5 MCS the anisotropy of the precipitate morphology is clear, and by $50 \mathrm{MCS}$ little further evolution of the now strongly anisotropic morphology may be observed. Unlike in the previous case where no applied stress is present, the precipitates here do not appear to significantly thicken with time. Instead, the precipitates appear to be evolving into straighter, more perfect needle-like structures. Such development of anisotropy in the shape of $\gamma^{\prime}$ precipitates under the influence of applied stress is a commonly observed $[10,11]$ and is known as rafting. These features may be seen more quantitatively in Fig. 11. Figure 11(b) shows that the overall coarsening rate, in terms of the mean precipitate size, increases significantly more slowly than in the stress-free case. This is supported by Fig. 11(a) where the fraction of broken bonds appears to saturate at a much higher level than in the stress-free case. The strong anisotropy in particle morphology may also be seen in that figure in terms of the small value of the ratio of broken bonds in the $y$-direction to those in the $x$-direction. The most striking similarity between the stress-free case and that for which the tensile stress is applied is the overall slow growth rate, compared to situations in which there is no net misfit [13].

Results analogous to those for the tensile loading case are found when the stress is compressive (see Figs 12 and 13), except that the rafting occurs in a directional orthogonal to the stress axis. The magnitude of the difference in the morphological anisotropy can be seen by comparing the ratio of broken bonds in the $y$ and $x$ directions for the two different loadings [compare Figs $11(\mathrm{a})$ and 13(a)].

\section{SUMMARY}

A specialized Monte Carlo microstructural lattice model, MCFET, has been developed which simulates 
1
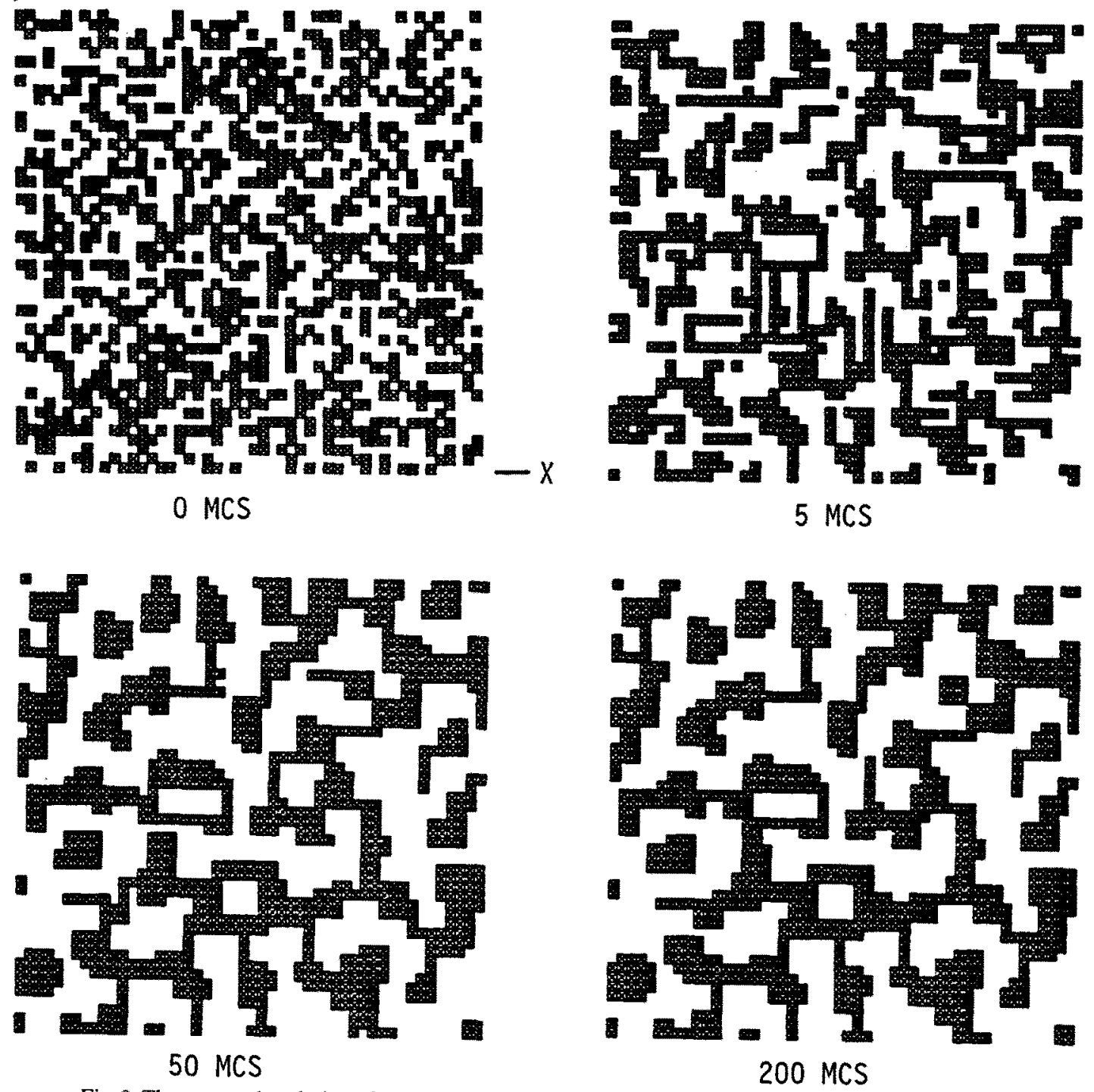

Fig. 8. The temporal evolution of a $(60-40 \%)$ two-phase microstructure with zero externally applied stress. The shaded sites correspond to the precipitate phase.
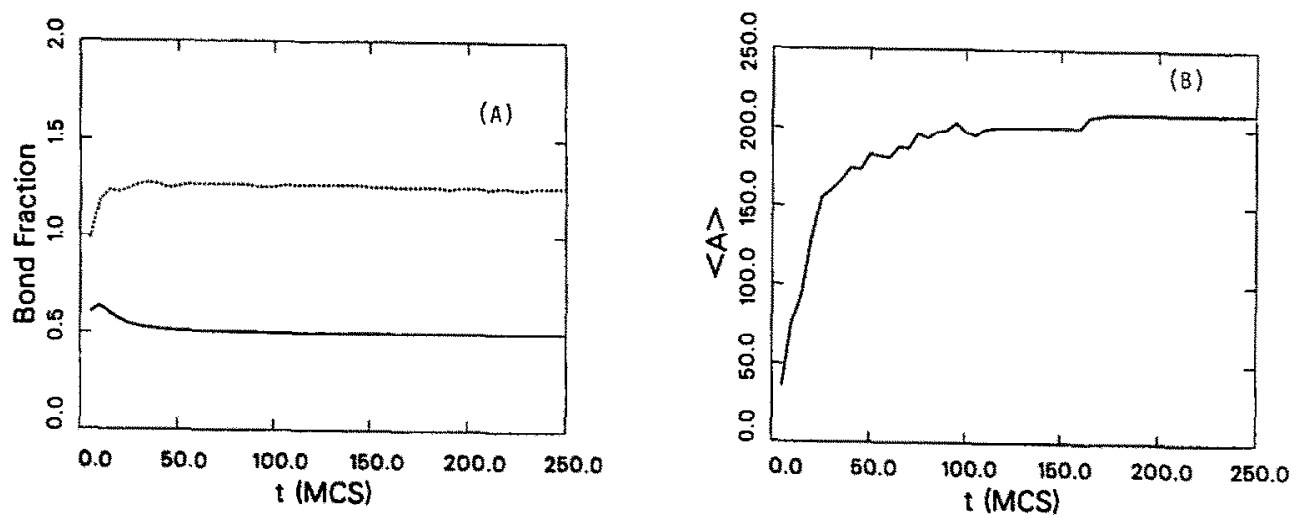

Fig. 9. (A) The fraction of bonds separating nearest neighbor sites of unlike phase as a function of time (solid line) averaged over five simulations under conditions identical to that shown in Fig. 8. The dotted line is the ratio of the number of broken bonds in the $y$-direction to those in the $x$-direction. (B) The time dependence of the mean precipitate size (area), $\langle A\rangle$. 


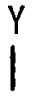
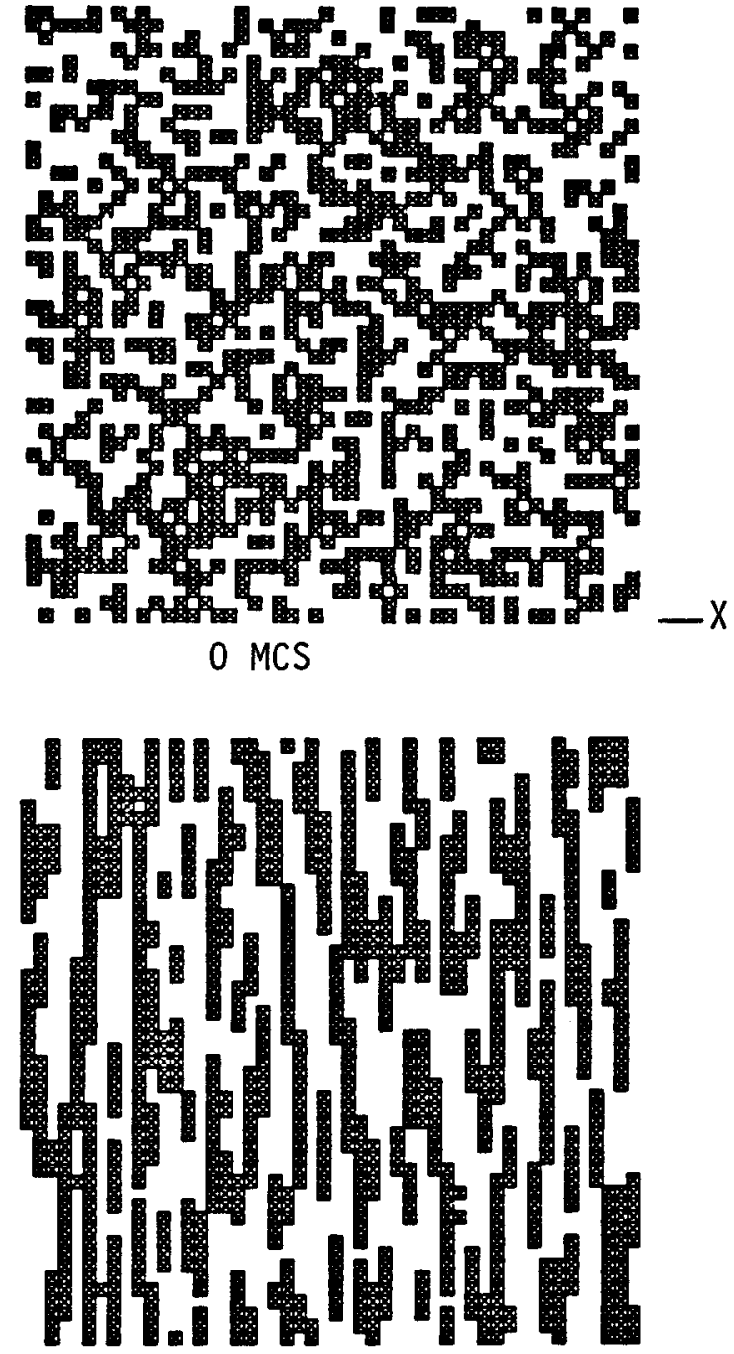

50 MCS

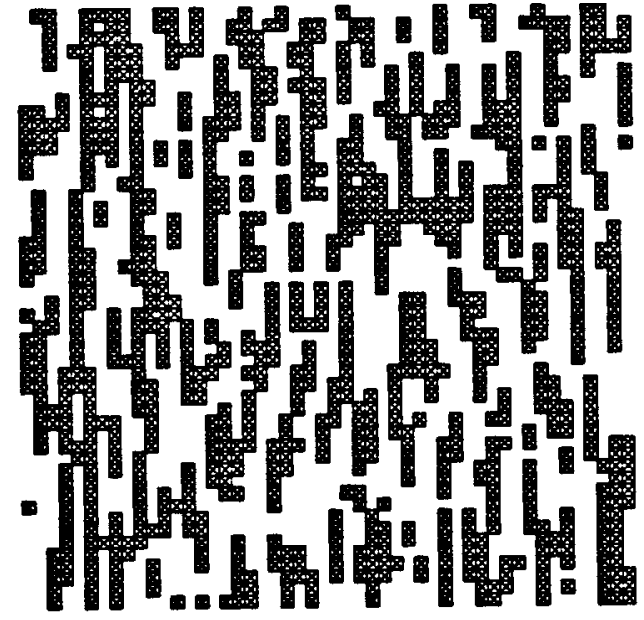

5 MCS

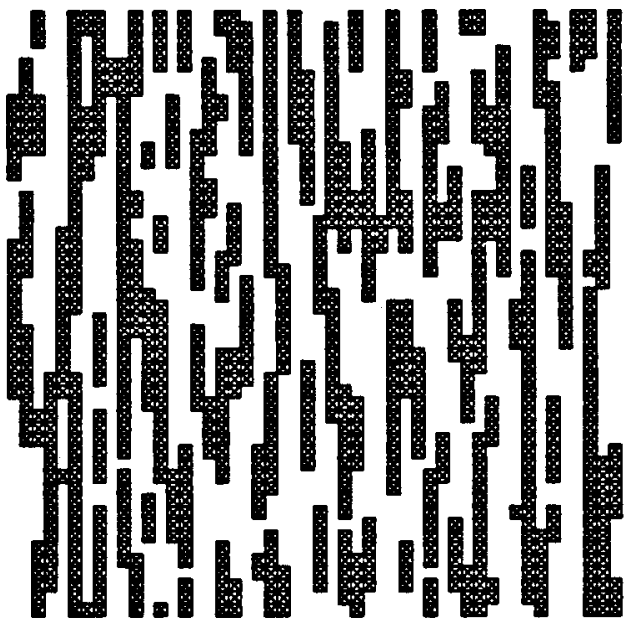

200 MCS

Fig. 10. Same as Fig. 8 but with a tensile stress in the $y$-direction.
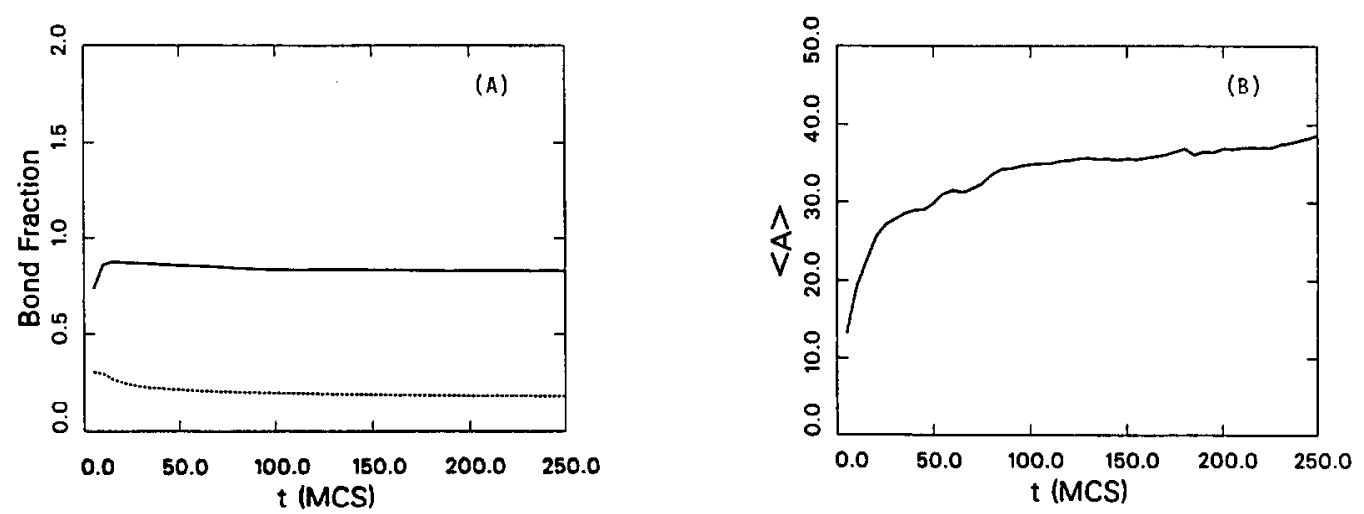

Fig. 11. Same as Fig. 9 but with a tensile stress in the $y$-direction. 
Y 4. 4. ror

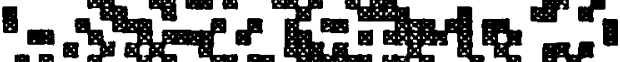

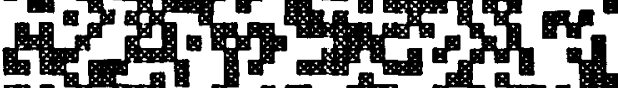

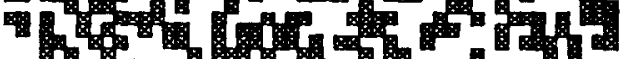

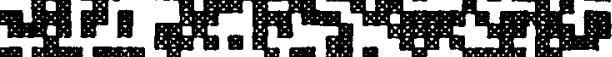

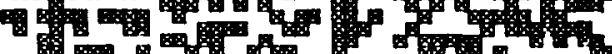

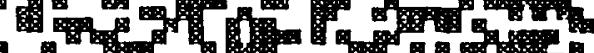

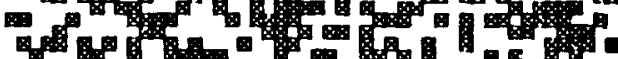
4800\% to bom

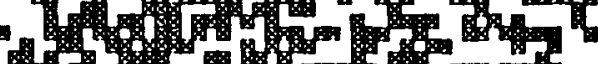
Fo 30

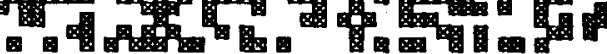
0 MCS

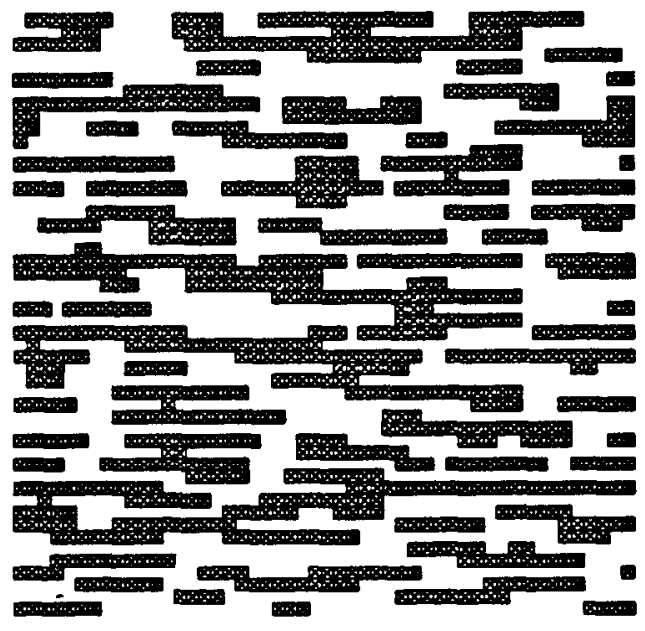
50 MCS
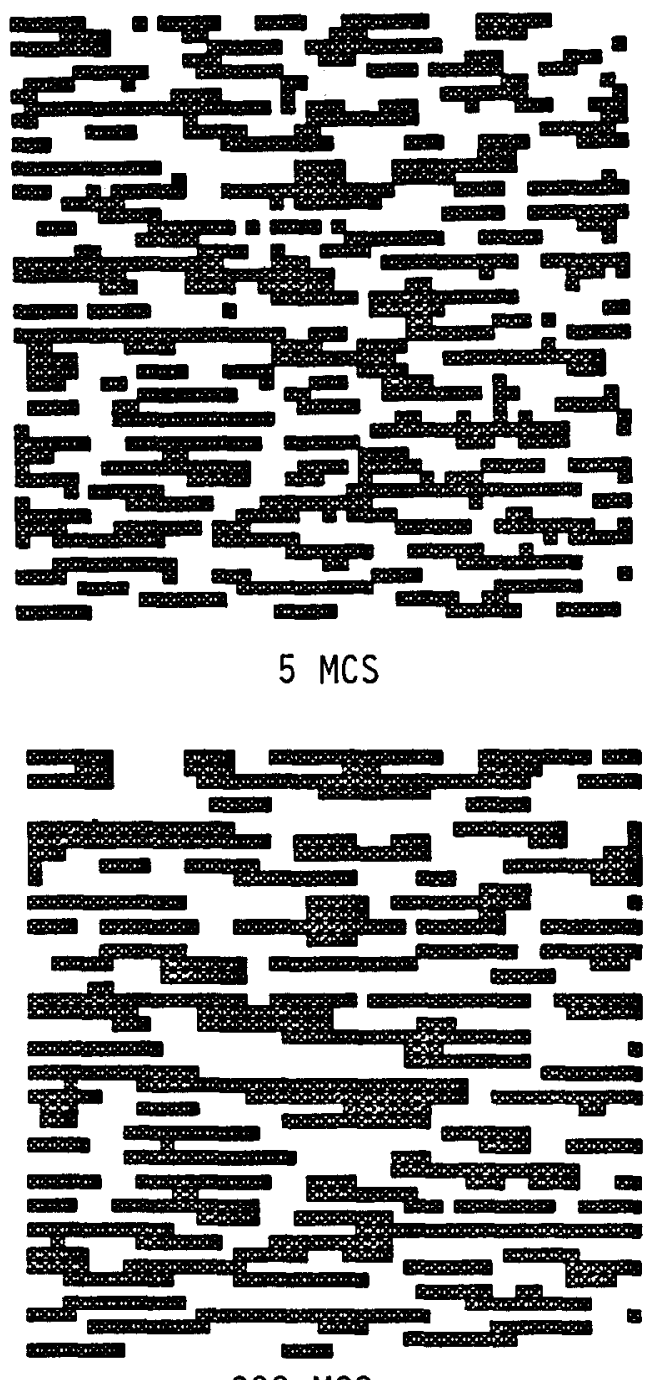

200 MCS

Fig. 12. Same as Fig. 8 but with a compressive stress in the $y$-direction.
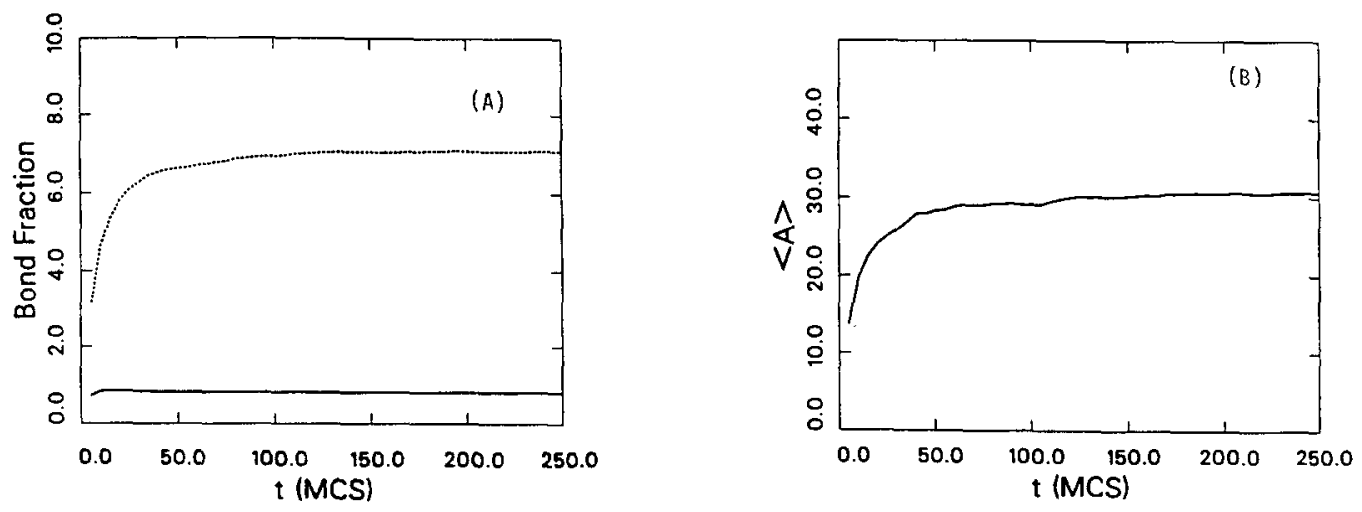

Fig. 13. Same as Fig. 9 but with a compressive stress in the $y$-direction. 
microstructural evolution in materials in which strain energy plays an important role in determining morphology. The elastic contribution to the energy of the system is estimated by way of a finite element procedure which is employed in the Monte Carlo procedure to determine the microstructural evolution. This model is currently capable of accounting for externally applied stresses, surface tension, misfit, elastic inhomogeneity, elastic anisotropies (cubic) and arbitrary temperatures.

The MCFET analysis has been validated by comparison with analytical calculations of the equilibrium morphologies of isolated particles in an infinite matrix. Larger simulations yield microstructures which are in good agreement with experimental observations (including the influence of applied stress).

Simulations on the coarsening of two-phase microstructures containing misfitting particles suggest that the presence of strong elastic interactions greatly decreases the rate of microstructural coarsening. When an external stress is applied, coarsening is further slowed. Even when coarsening is effectively stopped, further microstructural evolution occurs which tends to increase the perfection of the rafted/layered structures which form under the influence of an applied stress.
Acknowledgements-The authors would like to thank R. A. Mackay and A. D. Freed for helpful discussions on Ni-based superalloy behavior and elasticity theory, respectively.

\section{REFERENCES}

1. M. P. Anderson, D. J. Srolovitz, G. S. Grest and P. S. Sahni, Acta metall. 32, 783 (1984).

2. D. J. Srolovitz, M. P. Anderson, P. S. Sahni and G. S. Grest, Acta metall. 32, 793 (1984).

3. D. J. Srolovitz. M. P. Anderson and G. S. Grest, in Interface Migration and Control of Microstructure (edited by C. S. Pande, D. A. Smith, A. H. King and J. Walter), p. 155. Am. Soc. Metals, Metals Park, Ohio (1986).

4. K. Kawasaki, in Phase Transitions in Critical Phenomena (edited by C. Domb and M. S. Green), Vol. 2, p. 443. Academic Press, New York (1972).

5. L. J. Segerlind, Applied Finite Element Analysis. Wiley, New York (1976)

6. J. Gayda and D. J. Srolovitz, NASA Tech. Memo. 100215 (1987).

7. J. D. Eshelby, Proc. R. Soc. A241, 376 (1957).

8. A. Pineau, Acta metall. 24, 559 (1976).

9. W. C. Johnson and P. W. Voorhees, J. appl. Phys. 61, 1610 (1987).

10. R. A. MacKay and L. J. Ebert, Metall. Trans. A16, 1969 (1985).

11. C. Carry and J. L. Strudel, Acra metall. 26, 859 (1978).

12. T. Miyazaki, K. Nakamura and H. Mori, J. Mater. Sci. 14, 1827 (1979).

13. G. S. Grest and D. J. Srolovitz, Phys. Rev. B30, 5150 (1984). 